\title{
Myocardial extracellular volume assessed by cardiovascular magnetic resonance may predict adverse left ventricular remodeling in rheumatic heart disease after valvular surgery
}

\author{
Shuang Li ${ }^{1 \#}$, Simeng Wang ${ }^{1 \#}$, Jianqun Yu ${ }^{1}$, Jiayu Sun ${ }^{1}$, Wei Cheng ${ }^{1}$, Jing Liu ${ }^{1}$, Huaxia Pu ${ }^{1}$, Yucheng Chen ${ }^{2}$, \\ Liqing Peng ${ }^{1}$
}

${ }^{1}$ Department of Radiology, West China Hospital, Sichuan University, Chengdu, China; ${ }^{2}$ Department of Cardiology, West China Hospital, Sichuan University, Chengdu, China

Contributions: (I) Conception and design: S Li, J Yu, S Wang, Y Chen, L Peng; (II) Administrative support: J Sun, Y Chen, L Peng; (III) Provision of study materials or patients: J Sun, Y Chen, L Peng; (IV) Collection and assembly of data: S Wang, W Cheng, S Li; (V) Data analysis and interpretation: J Liu, H Pu, S Li; (VI) Manuscript writing: All authors; (VII) Final approval of manuscript: All authors.

"These authors contributed equally to this work.

Correspondence to: Liqing Peng, MD, PhD. Department of Radiology, West China Hospital, Sichuan University, No. 37 Guoxue Road, Chengdu 610041, China. Email: pengliqing@wchscu.cn; Yucheng Chen, MD, PhD. Department of Cardiology, West China Hospital, Sichuan University, No. 37 Guoxue Road, Chengdu 610041, China. Email: Chenyucheng2003@126.com.

Background: Only a few studies to date have focused on the application of cardiovascular magnetic resonance (CMR) in rheumatic heart disease (RHD); in particular, research on the application of T1mapping CMR sequences is limited. This study aimed to investigate whether diffuse myocardial fibrosis evaluated using preoperative T1 mapping and extracellular volume (ECV) fraction measurement could predict the progression of adverse left ventricular remodeling (LVR) after surgery.

Methods: A total of 32 adult patients with RHD and 30 healthy controls were recruited. Baseline clinical characteristics, CMR findings, and T1 mapping measurements were compared between the two groups. Transthoracic echocardiography measurements were collected before and after surgery. Patients with an increase in left ventricular end-diastolic volume of $>15 \%$ or a decrease in left ventricular ejection fraction of $>10 \%$ were classified into the adverse remodeling group; otherwise, patients were categorized into the nonadverse remodeling group.

Results: Compared with the healthy controls, patients with RHD had impaired biventricular function, enlarged ventricular volume, and increased native T1 and ECV values. Patients in the adverse remodeling group had higher ECV values than those in the non-adverse remodeling group $(33.25 \% \pm 3.67 \%$ vs. $28.45 \% \pm 4.46 \%, \mathrm{P}=0.002)$. Binary logistic regression analysis showed that the ECV value was associated with adverse LVR (odds ratio: 1.273, $\mathrm{P}=0.045$ ). $\mathrm{ECV}$ was found to be a sensitive biomarker for predicting adverse LVR (area under the curve: 0.78; sensitivity: 75.0\%; specificity: $77.3 \%$ ).

Conclusions: ECV has potential value for predicting the progression of adverse LVR and for identifying non-responders among patients with RHD undergoing surgery.

Keywords: Rheumatic heart disease (RHD); left ventricular remodeling (LVR); cardiac magnetic resonance (CMR); myocardial fibrosis; T1 mapping

Submitted Jun 28, 2021. Accepted for publication Dec 22, 2021; Published online: 10 Jan 2022.

doi: $10.21037 /$ qims-21-678

View this article at: https://dx.doi.org/10.21037/qims-21-678 


\section{Introduction}

Rheumatic heart disease (RHD) is caused by rheumatic fever, which occurs following oropharyngeal infection by hemolytic group A streptococcus (1). An estimated 33 million people are affected by rheumatic fever globally, and there are more than 400,000 new cases and over 230,000 deaths attributable to rheumatic fever or RHD each year, with South Asia, Africa, and the Pacific Islands particularly affected (2-4). According to previous studies, RHD is the dominant pathogenesis of multiple valvular diseases in both developed and developing countries $(5,6)$.

Myocardial fibrosis (MF) is considered to be a significant predictor of adverse outcomes in various cardiovascular diseases, such as nonischemic and dilated cardiomyopathy and aortic stenosis (7-9). Late gadolinium enhancement (LGE) in cardiac magnetic resonance (CMR) imaging facilitates the identification of focal MF, while T1 mapping by CMR imaging makes it possible to quantify diffuse MF in vivo (9-11). Developing countries account for most patients with RHD, but in recent years, rapid economic development has seen CMR imaging become widely available in these countries.

Left ventricular remodeling (LVR) is defined as the accommodative process of the left ventricle (LV) and can be measured by changes in cardiac morphology and function (12). This process is characterized by ventricular dilation, shape distortion, and wall hypertrophy (13). According to clinical observations, not all patients achieve regression of cardiac dilatation and ventricular ejection fraction recovery after successful valvular surgery, some patients even have a poor prognosis and may experience progression of adverse LVR (14). This study aimed to investigate whether diffuse MF evaluated with preoperative T1 mapping and extracellular volume (ECV) fraction measurement can be used to identify adverse LVR in patients with RHD undergoing surgery.

We present the following article in accordance with the STARD reporting checklist (available at https://qims. amegroups.com/article/view/10.21037/qims-21-678/rc).

\section{Methods}

\section{Study design and population}

Patients from West China Hospital were consecutively identified through the hospital database search for the period from 2013 to 2020 . The study was conducted in accordance with the Declaration of Helsinki (as revised in 2013), and was approved by West China Hospital's ethics board (No. 2019-756). The requirement to obtain individual consent for this analysis was waived due to its retrospective nature.

The inclusion criteria for patients in this study were as follows: (I) clinically diagnosed with RHD; (II) had undergone comprehensive preoperative CMR; and (III) had undergone pre- and postoperative echocardiography. The exclusion criteria were as follows: (I) patients with other organic heart diseases (based on history, or echocardiography or CMR findings), or with a history of cardiac surgery; and (II) patients with poor-quality CMR images that were inadequate for analysis.

Baseline demographic and clinical data of all the included patients were collected. Also, a group of healthy volunteers who underwent CMR were enrolled as healthy controls (HCs).

\section{CMR scanning protocol and imaging analysis}

All study participants underwent CMR on a 3.0-T MRI scanner (Magnetom Skyra or Tim Trio; Siemens Medical Solutions, Erlangen, Germany). Following the acquisition of localizers, balanced, steady-state free precession (SSFP) cine images were obtained in 8-12 matching short-axis and 3 radial long-axis planes (3-, 4-chamber, and LV 2-chamber views). The cardiac frame number of each short-axis plane was 25 . The parameters for cine imaging were as follows: field of view $(\mathrm{FoV}), 250 \mathrm{~mm} \times 300 \mathrm{~mm}$; matrix size, $208 \times 139$ pixels; integrated parallel acquisition technique (iPAT), 2; repetition time (TR), $3.3 \mathrm{~ms}$; echo time (TE), $1.22 \mathrm{~ms}$; slice thickness, $8 \mathrm{~mm}$; and, flip angle, $40^{\circ}$.

T1 mapping was performed using modified Look-Locker inversion recovery (MOLLI) imaging. The parameters for MOLLI were as follows: TR $346.56 \mathrm{~ms}$, TE $1.22 \mathrm{~ms}$, thickness $8 \mathrm{~mm}$, and flip angle 35 degrees. Contrast media, $0.5 \mathrm{mmol} / \mathrm{mL}$ gadobenate dimeglumine (MultiHance, Bracco, Milan, Italy) was injected at a dose of $0.1 \mathrm{~mL} / \mathrm{kg}$ body weight with $20 \mathrm{~mL}$ saline solution at a flow rate of $3.0 \mathrm{~mL} / \mathrm{s}$ at first, and then at a dose of $0.05 \mathrm{~mL} / \mathrm{kg}$ body weight with $20 \mathrm{~mL}$ saline solution after the perfusion images were acquired. The LGE images were acquired 10 to 15 minutes after administration of the contrast media.

All image data were uploaded to the dedicated cardiac MRI post-processing software cvi42 (version 5.11.3, Circle Cardiovascular Imaging, Inc., Calgary, Canada). The endocardial and epicardial borders of the left and right ventricles were traced in short-axis slices, and the left 
and right ventricular end-systolic volumes (LVESV and RVESV, respectively), end-diastolic volumes (LVEDV and RVEDV, respectively), and ejection fractions (LVEF and RVEF, respectively) were calculated automatically. Native $\mathrm{T} 1$ and post-contrast $\mathrm{T} 1$ values were measured in the whole myocardium and a region of interest (ROI) was manually traced in the septal myocardium of the left ventricular basal and middle segments. Finally, the average T1 values and ECV fractions were acquired. The ECV was calculated from native and post-contrast T1 mapping values using the following formula (15):

$$
\text { ECV }=(1-\text { hematocrit }) \frac{\left(\frac{1}{T 1_{\text {myo post }}}-\frac{1}{T 1_{\text {myo pre }}}\right)}{\left(\frac{1}{T 1_{\text {blood post }}}-\frac{1}{T 1_{\text {blood pre }}}\right)}
$$

The hematocrit was derived from the routine blood test closest in time to the CMR examination. The borders and ROIs were traced manually by two radiologists, respectively.

\section{Transthoracic echocardiography (TTE) and follow-up}

All participants underwent TTE before and after surgery. The average follow-up duration was 16.7 months (range, 1-60 months). If a patient had undergone several postoperative examinations, the latest one was used in our study. Patients in this study were retrospectively recruited from the hospital's imaging database. Their LVEF, LVESV, and LVEDV before and after surgery were collected from the electronic records. TTE was performed on a Philips 7500, Philips IE 33, or Philips EPIQ 7C system (Philips Ultrasound System, the Netherlands). The ultrasonic probe was $X 7-2 t$, and the frequency ranged from 2 to $7 \mathrm{MHz}$. Color Doppler was used to assess valvular stenosis or regurgitation. If a patient had ventricular chamber distortion and dilation, the LVEF was measured using the biplane Simpson's method.

Many researchers have studied LVR across a wide range of diseases, among which the definition of LVR differs. Olsen et al. (16) defined patients with an increase in LVEDV of $>15 \%$ or a decrease in LVEF of $>10 \%$ as being in progression. Legallois et al. (17), who reviewed 37 studies involving 4,209 patients (from January 2010 to August 2019), proposed that an increase in LVESV of $12 \%$ to $15 \%$ and an increase in LVEDV of $12 \%$ to $20 \%$ might be the optimal criterion or defining adverse LVR in patients with myocardial infarction. In our study, patients with an increase in LVEDV of $>15$ or a decrease in LVEF of $>10 \%$ were categorized into the adverse remodeling group; otherwise, patients were assigned to the non-adverse remodeling group.

\section{Statistical analysis}

Continuous variables were expressed as mean \pm standard deviation. Differences in continuous variables were analyzed using the Mann-Whitney U-test or Student's $t$-test. An intraclass correlation coefficient (ICC) was used to measure the reliability of ratings in CMR measurement. Linear correlation analyses were performed to evaluate the relationships between ECV and postoperative LVESV, LVEDV, and LVEF. Binary logistic regression was performed to identify the predictors of LVR. Receiver operating characteristic (ROC) analysis was performed to determine whether ECV can be used to differentiate adverse LVR from reverse LVR. Statistical analyses were performed using the SPSS software v. 19.0 (SPSS Inc., Chicago, IL, USA).

\section{Results}

\section{Clinical manifestations of the patients with RHD}

The process of patient selection is shown in the flow diagram in Figure 1. A total of 89 patients were diagnosed with RHD and underwent CMR for the period from 2013 and to 2020. Patients were excluded from the study due to having the following conditions: congenital heart disease $(\mathrm{n}=2$, including 1 case of tetralogy of Fallot and 1 case of foramen ovale), infective endocarditis $(n=1)$, dilated cardiomyopathy $(\mathrm{n}=1)$, and coronary heart disease $(\mathrm{n}=1)$. Thirteen outpatients were also excluded. Of 71 hospitalized patients, 58 underwent valvular surgery, 13 patients did not undergo surgery (including 8 high-risk patients, 4 patients who refused surgery, and 1 patient without indication for surgery). All 58 patients who underwent surgery had preoperative TTE; in 46 cases, the TTE was performed in our institution. Among these 46 patients, 32 patients underwent preoperative T1 mapping, and their cardiac function was also measured. These 32 patients (scanned between October 2013 and May 2019) were included in the study.

A total of 30 healthy volunteers (mean age, $48.40 \pm 14.08$ years; range, $35-65$ years; male, $n=12$; female, $\mathrm{n}=18$ ) were enrolled as HCs. The demographic data of all participants were collected (including age, sex, height, 


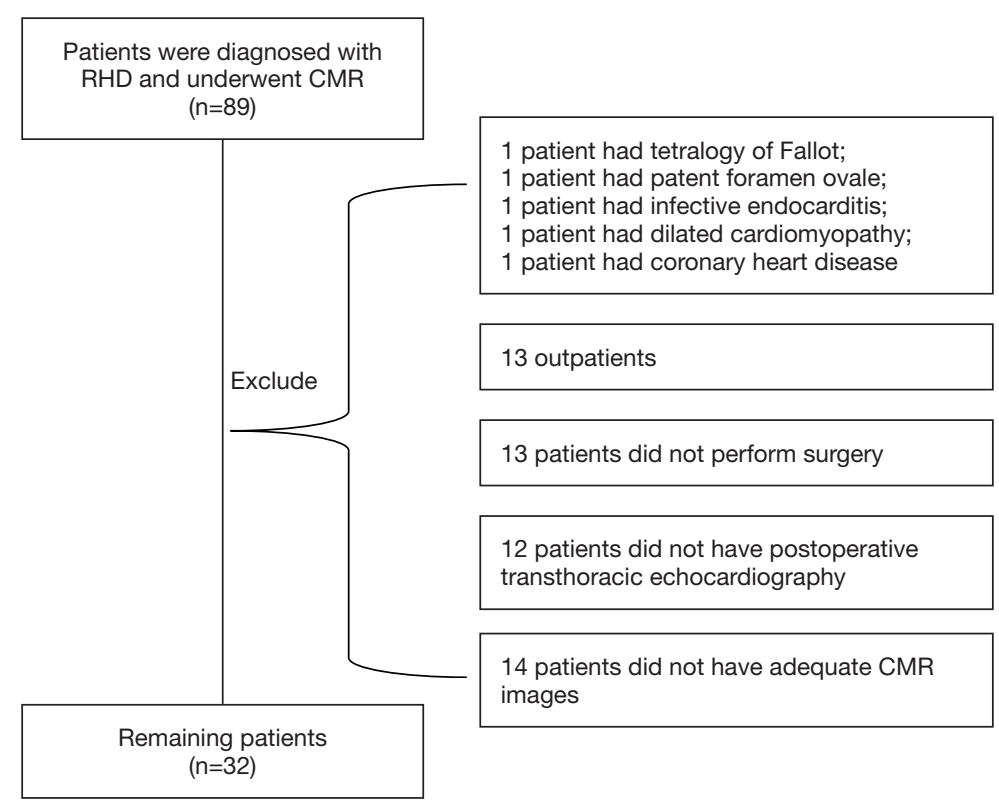

Figure 1 Flow diagram of the study population. RHD, rheumatic heart disease; CMR, cardiovascular magnetic resonance.

weight, blood pressure, and heart rate) together with their CMR imaging findings.

All patients had multiple or mixed valvular diseases. All 32 patients were symptomatic, and most of them complained of chest tightness or tightness in breathing $(26 / 32,81.3 \%)$. Lower extremity edema (4/32, $12.5 \%)$, abdominal distension $(3 / 32,9.4 \%)$, cough or dry cough $(4 / 32,12.5 \%)$, palpitations $(4 / 32,12.5 \%)$, dizziness (1/32, $3.1 \%)$, and chest pain $(1 / 32,3.1 \%)$ were also reported. Some patients experienced multiple clinical symptoms simultaneously. The duration of clinical symptoms ranged from 1 to 360 months (average time, 67.5 months). A total of $28(87.5 \%)$ patients presented with atrial fibrillation. The majority of patients $(22 / 32,68.8 \%)$ were categorized as New York Heart Association (NYHA) class III, 9 (28.1\%) patients were categorized as class II, and 1 patient was categorized as class IV (18).

Valvular surgery procedures received by the patients included the following: mitral valve replacement (MVR) and tricuspid valve valvuloplasty (TVP) $(18 / 32,56.3 \%)$; aortic valve replacement (AVR), MVR, and TVP (6/32, 18.8\%); MVR and AVR (2/32, 6.3\%); MVR and tricuspid valve replacement (TVR) (1/32, 3.1\%), MVR alone (1/32, 3.1\%); TVR alone (1/32, 3.1\%); MVR, TVR, and AVR (1/32, $3.1 \%)$; MVR, TVP, and pulmonary valve valvuloplasty (1/32, 3.1\%); and MVR, pulmonary valve replacement, and $\operatorname{TVP}(1 / 32,3.1 \%)$.

\section{Comparison of different methods for evaluating $T 1$ values}

As shown in Figure 2, native T1 and post-contrast T1 values were measured in two different ways. There was no statistically significant difference in the native $\mathrm{T} 1$ or post-contrast $\mathrm{T} 1$ values of the whole myocardium and septal myocardium in patients with RHD (native T1: $1,308.54 \pm 79.58$ vs. $1,303.19 \pm 79.67 \mathrm{~ms}, \mathrm{P}=0.39$; post contrast T1: $481.58 \pm 61.40$ vs. $486.07 \pm 82.39 \mathrm{~ms}, \mathrm{P}=0.47$ ). Also, the interobserver reproducibility was good, with ICC values ranging from 0.893 to 0.938 (all $\mathrm{P}<0.05$ ). The values measured in the septal myocardium were used in this study.

\section{Comparisons of patients with RHD and HCs}

The demographic data and CMR findings of the patients with RHD and HCs were compared (Table 1). No differences in demographic data were observed. Patients with RHD had significantly reduced ejection fraction, enlarged ventricular volume, and higher left ventricular mass (all $\mathrm{P}<0.05$ ). Regarding $\mathrm{T} 1$ mapping parameters, the RHD group had statistically significantly higher native T1 (RHD group vs. HCs: $1,306.34 \pm 83.23$ vs. $1,210.15 \pm 46.33 \mathrm{~ms}, \mathrm{P}<0.001)$ and $\mathrm{ECV}$ (RHD group vs. HCs: $30.25 \% \pm 4.56 \%$ vs. $27.95 \% \pm 3.14 \%, \mathrm{P}=0.024)$ values than did the HCs. 

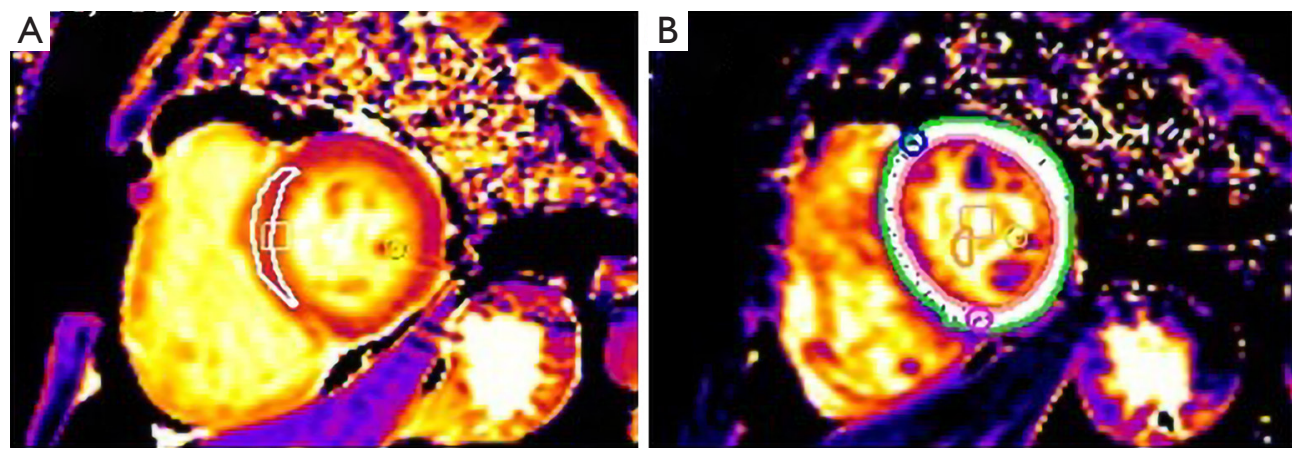

Figure 2 Different measurement methods of T1 mapping. Native T1 and post-contrast T1-values were measured by manually tracing a region of interest in the septal myocardium of the left ventricular basal and middle segments (A) and the whole myocardium (B).

Table 1 Comparisons of patients with RHD and healthy controls

\begin{tabular}{|c|c|c|c|}
\hline Parameters & Control group $(n=30)$ & RHD group $(n=32)$ & $P$ value \\
\hline Male, n [\%] & $12[40]$ & 8 [25] & 0.207 \\
\hline Age (years) & $48.40 \pm 14.08$ & $51.72 \pm 12.89$ & 0.337 \\
\hline $\mathrm{BMI}\left(\mathrm{kg} / \mathrm{m}^{2}\right)$ & $22.97 \pm 2.58$ & $25.33 \pm 6.12$ & 0.102 \\
\hline $\mathrm{SBP}(\mathrm{mmHg})$ & $119.21 \pm 9.27$ & $118.22 \pm 14.34$ & 0.771 \\
\hline $\mathrm{DBP}(\mathrm{mmHg})$ & $71.70 \pm 5.00$ & $75.59 \pm 13.28$ & 0.137 \\
\hline \multicolumn{4}{|l|}{ Cardiac function } \\
\hline CMR-LVEF (\%) & $63.45 \pm 4.78$ & $40.41 \pm 12.53$ & $<0.001$ \\
\hline CMR-RVEF (\%) & $56.93 \pm 7.64$ & $37.19 \pm 9.50$ & $<0.001$ \\
\hline CMR-RVESV (mL) & $43.91 \pm 12.12$ & $96.75 \pm 42.26$ & $<0.001$ \\
\hline CMR-RVEDV (mL) & $119.43 \pm 22.97$ & $155.80 \pm 72.02$ & 0.002 \\
\hline LV mass $(\mathrm{g})$ & $73.99 \pm 18.19$ & $96.96 \pm 44.99$ & $<0.001$ \\
\hline \multicolumn{4}{|l|}{ T1 mapping } \\
\hline Native T1 (ms) & $1,210.15 \pm 46.33$ & $1,306.34 \pm 83.23$ & $<0.001$ \\
\hline Post-contrast T1 (ms) & $472.34 \pm 41.23$ & $511.39 \pm 65.13$ & 0.07 \\
\hline ECV (\%) & $27.95 \pm 3.14$ & $30.25 \pm 4.56$ & 0.024 \\
\hline
\end{tabular}

Data are expressed as mean \pm standard deviation if not otherwise specified. RHD, rheumatic heart disease; BMI, body mass index; SBP, systolic blood pressure; DBP, diastolic blood pressure; CMR, cardiac magnetic resonance; LVEF/RVEF, left/right ventricular ejection fraction; LVESV/RVESV, left/right ventricular end-systolic volume; LVEDV/RVEDV, left/right ventricular end-diastolic volume; LV, left ventricular; ECV, extracellular volume fraction. 
Table 2 Pre- and postoperative TTE findings of patients with RHD

\begin{tabular}{|c|c|c|c|}
\hline Parameters & Non-adverse remodeling $(n=20)$ & Adverse remodeling $(n=12)$ & $P$ value \\
\hline$T T E_{\text {-pre }}$ & $55.95 \pm 10.72$ & $55.25 \pm 10.72$ & 0.84 \\
\hline TTE- ${ }_{\text {post }}$ & $63.00 \pm 7.34$ & $50.25 \pm 12.85$ & 0.01 \\
\hline \multicolumn{4}{|l|}{ LVESV (mL) } \\
\hline TTE- & $40.00 \pm 28.17$ & $62.64 \pm 23.55$ & 0.03 \\
\hline \multicolumn{4}{|l|}{ LVEDV (mL) } \\
\hline TTE- & $117.37 \pm 61.11$ & $121.60 \pm 40.47$ & 0.85 \\
\hline TTE- ${ }_{\text {post }}$ & $101.11 \pm 40.88$ & $126.27 \pm 29.57$ & 0.09 \\
\hline
\end{tabular}

Data are expressed as mean \pm standard deviation. TTE, transthoracic echocardiography; RHD, rheumatic heart disease; TTE-pre, preoperative TTE; TTE-post, postoperative TTE; LVEF, left ventricular ejection fraction; LVESV, left ventricular end-systolic volume; LVEDV, left ventricular end-diastolic volume.

\section{Pre-and postoperative TTE findings of patients with RHD}

Postoperative TTE-LVESV (non-adverse remodeling $v s$. adverse remodeling: $40.00 \pm 28.17$ vs. $62.64 \pm 23.55 \mathrm{~mL}$; $\mathrm{P}=0.03$ ) and TTE-LVEF (non-adverse remodeling $v s$. adverse remodeling: $63.00 \% \pm 7.34 \%$ vs. $50.25 \% \pm 12.85 \%$, $\mathrm{P}=0.01$ ) were statistically significantly different between patients with non-adverse remodeling and adverse remodeling (Table 2).

\section{Predictors of post-operative adverse LVR}

The clinical characteristics, CMR findings, and T1 mapping parameters of patients with non-adverse remodeling and adverse remodeling were compared (Table 3). Preoperative parameters, including CMR-LVEDV, CMR-RVESV, CMR-RVEDV, and ECV $(\mathrm{P} \leq 0.10)$, were assessed by binary logistic regression analysis (Table 4). ECV was the only variable found to be associated with adverse $\mathrm{LVR}(\mathrm{P}=0.045$, odds ratio: 1.273 , 95\% CI: 1.001-1.604). Two representative cases are presented in Figure 3.

\section{ROC curve analysis}

ROC curve analysis showed that $30.5 \%$ was the optimal ECV cutoff value to identify patients with adverse LVR (sensitivity: 75.0\%; specificity: 77.2\%; AUC: 0.78) (Figure 4).

\section{Discussion}

This study investigated the MF in chronic RHD assessed by $\mathrm{T} 1$ mapping, and explored the significance of $\mathrm{MF}$ in predicting postoperative outcomes. The main findings are that diffuse MF can exist in patients with RHD and that an ECV of $\geq 30.5 \%$ may predict the progression of adverse LVR after valvular surgery, with a high sensitivity of $75.0 \%$ and a specificity of $77.3 \%$.

MF is a common finding in patients with RHD. It is secondary to abnormal hemodynamics caused by valvular diseases and is the sequelae of the chronic inflammatory process of rheumatic fever (19-21). As a pathophysiological mechanism of cardiac structural and functional changes, MF has been reported to be associated with LV dysfunction, heart failure, and a poor prognosis (22). Diffuse MF results from collagen deposition and myofibroblastic activity in the early stages of valvular disease, which may lead to ventricular wall stiffness and, ultimately, left ventricular decompensation. Therefore, monitoring the changes of diffuse MF is especially important.

Endomyocardial biopsy is an established gold standard for MF detection and quantification (23). However, this procedure is uncommonly used in clinical practice due to its invasiveness and sampling bias (9). LGE imaging can identify focal MF, and T1 mapping imaging can quantify diffuse MF, noninvasively (10). Studies have also confirmed an excellent correlation between measurement of MF by histopathology and CMR (7). The ECV was calculated by combining native and post-contrast $\mathrm{T} 1$, and hematocrit reflecting an expanded extracellular matrix (15). Previously, Monti et al. demonstrated that contrast-enhanced computed tomography also has potential value for assessing ECV (24). 
Table 3 Baseline and CMR findings of patients with different outcomes

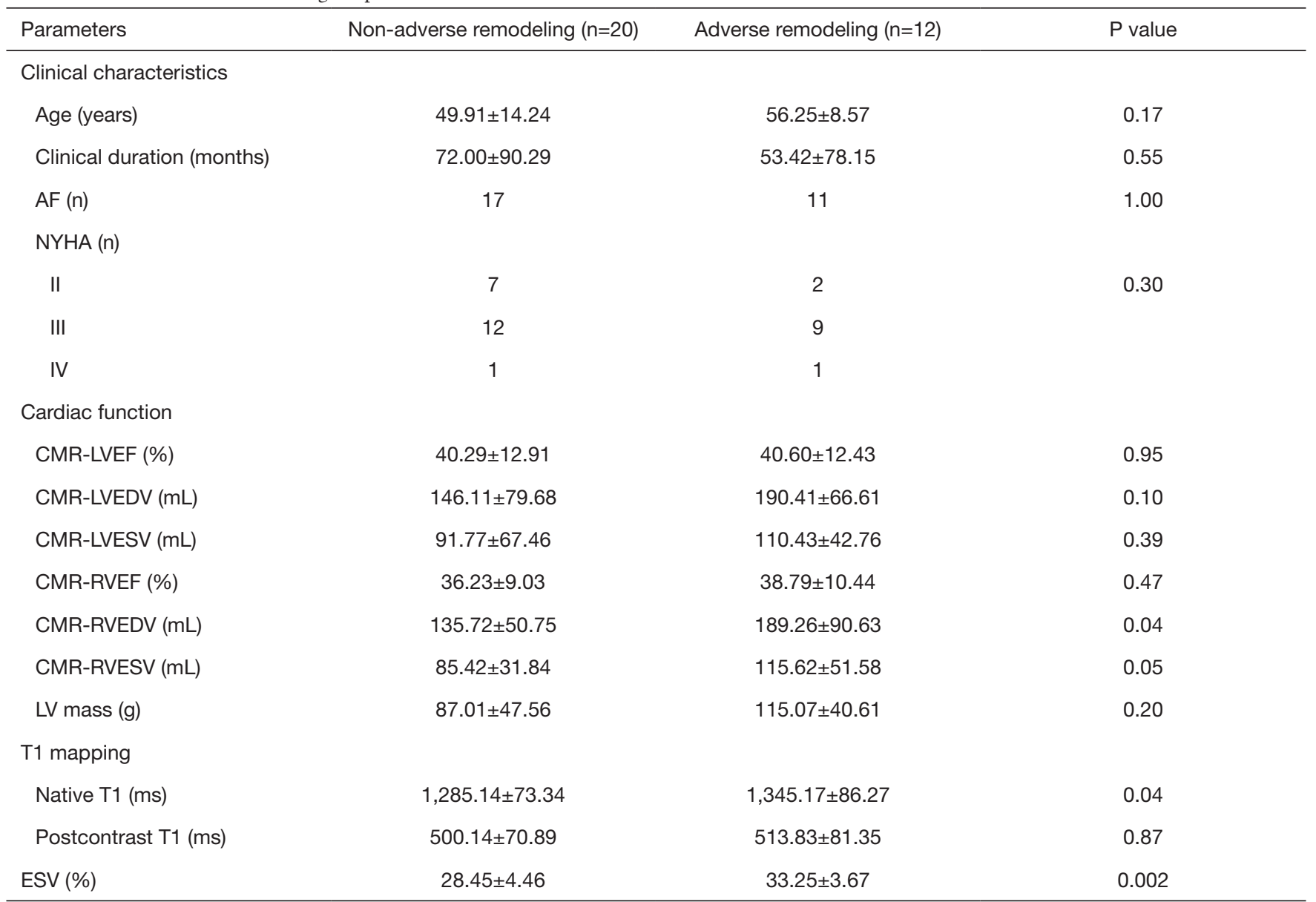

Data are expressed as mean \pm standard deviation if not otherwise specified. AF, atrial fibrillation; NYHA, New York Heart Association; CMR, cardiac magnetic resonance; LVEF/RVEF, left/right ventricular ejection fraction; LVESV/RVESV, left/right ventricular end-systolic volume; LVEDV/RVEDV, left/right ventricular end-diastolic volume; LV, left ventricular; ECV, extracellular volume fraction.

Table 4 Binary logistic regression analysis of independent predictors of outcomes

\begin{tabular}{lll}
\hline Variables & OR $(95 \% \mathrm{Cl})$ & P value \\
\hline CMR-LVEDV $(\mathrm{mL})$ & $1.003(0.991-1.015)$ & 0.64 \\
CMR-RVESV $(\mathrm{mL})$ & $1.012(0.946-1.083)$ & 0.73 \\
CMR-RVEDV $(\mathrm{mL})$ & $1.002(0.963-1.043)$ & 0.91 \\
ECV $(\%)$ & $1.273(1.001-1.604)$ & 0.045 \\
\hline
\end{tabular}

OR, odds ratio; $\mathrm{Cl}$, confidence interval; CMR, cardiac magnetic resonance; LVEDV/RVEDV, left/right ventricular end-diastolic volume; RVESV, right ventricular end-systolic volume; ECV, extracellular volume fraction.
In recent years, researchers have reported on the application of T1 mapping technology in non-rheumatic valvular diseases, such as aortic stenosis, and aortic and mitral regurgitation. Dusenbery et al. (25), for instance, demonstrated that young patients with congenital aortic stenosis had higher ECV than HCs. Furthermore, T1 mapping measurements also hold promise for predicting clinical outcomes. A multicenter study of 440 patients undergoing valve replacement for moderate aortic stenosis found that the ECV value was not only associated with cardiovascular mortality but was also independently associated with all-cause mortality (26). However, the value 


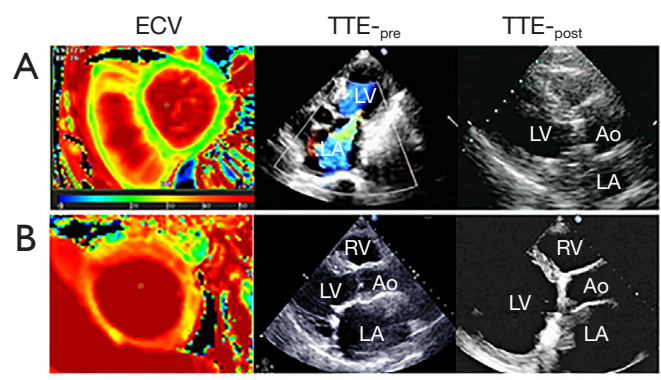

\begin{tabular}{ccccc}
\hline Patients & TTE & LVESD $(\mathrm{mm})$ & LVEDD $(\mathrm{mm})$ & LVEF (\%) \\
\hline Patient 1 & TTE- pre & 41 & 62 & 63 \\
ECV (27.64\%) & TTE- post & 34 & 44 & 61 \\
Patient 2 & TTE- pre & 41 & 60 & 58 \\
ECV (36.88\%) & TTE- & 58 & 77 & 34 \\
\hline
\end{tabular}

Figure 3 Representative images of two patients with different ECV values. Patient 1 (A) is a 65 -year-old male with a low ECV value $(27.64 \%)$, and patient 2 (B) is a 55-year-old man with a high ECV value (36.88\%). Both patients underwent aortic valve replacement, mitral valve replacement, and tricuspid valvuloplasty. Patient 2 was rehospitalized for heart failure 2 years after surgery. TTE, transthoracic echocardiography; LV, left ventricle; LA, left atrium; RV, right ventricle; RA, right atrium; Ao, aortic; ECV, extracellular volume fraction; LVESD, left ventricular end systolic dimension; LVEDD, left ventricular end diastolic dimension; LVEF, left ventricular ejection fraction.

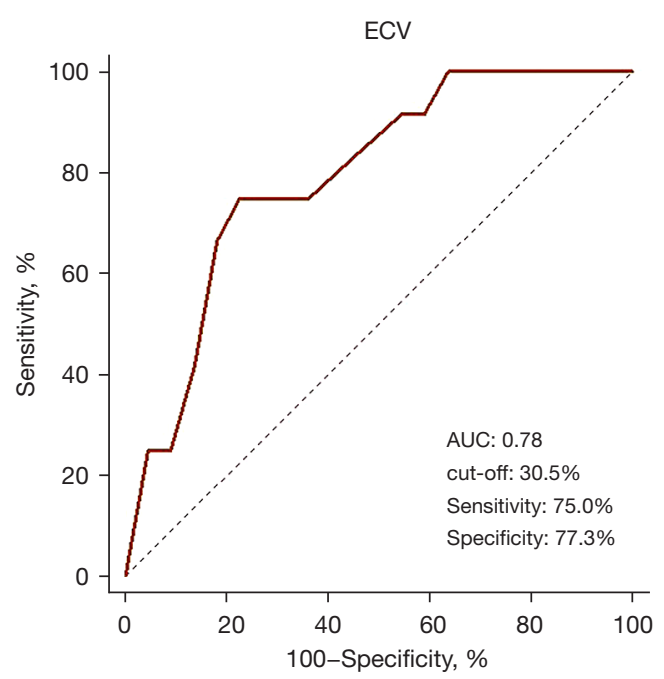

Figure 4 ROC analysis to differentiate adverse LVR from nonadverse LVR. In ROC analysis, the sensitivity and specificity of ECV for differentiating the adverse LVR in patients with RHD are $75.0 \%$ and $77.3 \%$, respectively, and the optimal cutoff value of ECV for identification of adverse LVR is $30.5 \%$. ROC, receiver operating characteristic; AUC, area under the curve; LVR, left ventricular remodeling; ECV, extracellular volume fraction; RHD, rheumatic heart disease.

of ECV in patients with rheumatic valvular disease has not been well studied so far.

Previous CMR findings in RHD have mainly focused on the pathophysiologic changes of valves and cardiac function (27). Only a few published studies have evaluated MF in patients with RHD using CMR, with most studies on this topic presented as case reports or short case series.
Shriki et al. (28) reported on 3 patients with LGE in the atrial wall, and Meel et al. reported on 21 patients with chronic rheumatic mitral regurgitation, 4 of whom had evidence of LGE in the LV (29). A study of 47 patients with mitral stenosis found that LGE was associated with postoperative morbidity following mitral valve surgery (30). To our knowledge, ours is the first study to comprehensively evaluate T1 mapping measurements in the septal and whole myocardium using a 3T MR system.

In our study, all patients had multiple or mixed valvular diseases. Patients with heterogeneous valve conditions were included, despite the potential differences in $\mathrm{LV}$ volume overload and pressure overload. Isolated cardiac valve affection is unusual in rheumatic valvular disease, especially in patients at the advanced stages of disease. Although more than a third of patients who present for surgery have more than one valve affected (31), single valve disease (regurgitation or stenosis) has received the most attention in published studies $(32,33)$. Due to this lack of data, there are no evidence-based recommendations for the timing of surgery in mixed or multiple valve disease (32).

In our preliminary study, we used CMR to evaluate patients with RHD to promote precision treatment. The results hint that patients with RHD who have more diffuse MF preoperatively have an increased risk of experiencing adverse LVR than those who do not. We speculate that the stiffness of the ventricular wall may increase with the accumulation of myocardial interstitial fibrosis, manifesting as a rise in the ECV value. As the ECV increases, the ventricular elasticity may decompensate, making LVEF recovery impossible after correction of hemodynamic abnormality with valvular surgery in patients with RHD. 
Hence, a more intensive follow-up plan could be deployed to prevent fatal cardiac complications in these patients. Future investigations should focus on whether diffuse MF evaluated based on the ECV can help to monitor myocardial change early and identify the optimal time for surgery.

Our study has several limitations. Firstly, the limited number of patients may result in a type II error. Secondly, it would have been better if the patients had undergone postoperative CMR to assess the changes of MF in patients before and after surgery. Thirdly, the patients with RHD who were included had mixed or multiple valvular diseases. Despite the heterogeneity of valvular etiology of the study, the ECV value gave a moderate performance for predicting adverse LVR in patients with RHD following surgery. Finally, because many patients were lost to follow-up, our study could not focus on long-term clinical outcomes, such as death or rehospitalization.

\section{Conclusions}

ECV may be able to predict the progression of adverse LVR and identify non-responders among patients with RHD undergoing surgery. Studies with a larger sample size are needed in the future.

\section{Acknowledgments}

Funding: This work was supported by the National Natural Science Foundation of China (No. 81601462), the Key Research \& Development Project of Science and Technology of Sichuan Province (No. 2021YFS0142), and 1.3.5 Project for Disciplines of Excellence, West China Hospital, Sichuan University (No. ZYGD1801).

\section{Footnote}

Reporting Checklist: The authors have completed the STARD reporting checklist. Available at https://qims.amegroups. com/article/view/10.21037/qims-21-678/rc

Conflicts of Interest: All authors have completed the ICMJE uniform disclosure form (available at https://qims. amegroups.com/article/view/10.21037/qims-21-678/coif). The authors have no conflicts of interest to declare.

Ethical Statement: The authors are accountable for all aspects of the work in ensuring that questions related to the accuracy or integrity of any part of the work are appropriately investigated and resolved. The study was conducted in accordance with the Declaration of Helsinki (as revised in 2013), and was approved by West China Hospital's ethics board (No. 2019-756). The requirement to obtain individual consent for this analysis was waived due to its retrospective nature.

Open Access Statement: This is an Open Access article distributed in accordance with the Creative Commons Attribution-NonCommercial-NoDerivs 4.0 International License (CC BY-NC-ND 4.0), which permits the noncommercial replication and distribution of the article with the strict proviso that no changes or edits are made and the original work is properly cited (including links to both the formal publication through the relevant DOI and the license). See: https://creativecommons.org/licenses/by-nc-nd/4.0/.

\section{References}

1. Liu M, Lu L, Sun R, Zheng Y, Zhang P. Rheumatic Heart Disease: Causes, Symptoms, and Treatments. Cell Biochem Biophys 2015;72:861-3.

2. Watkins DA, Johnson CO, Colquhoun SM, Karthikeyan G, Beaton A, Bukhman G, Forouzanfar MH, Longenecker CT, Mayosi BM, Mensah GA, Nascimento BR, Ribeiro ALP, Sable CA, Steer AC, Naghavi M, Mokdad AH, Murray CJL, Vos T, Carapetis JR, Roth GA. Global, Regional, and National Burden of Rheumatic Heart Disease, 1990-2015. N Engl J Med 2017;377:713-22.

3. Carapetis JR, Steer AC, Mulholland EK, Weber M. The global burden of group A streptococcal diseases. Lancet Infect Dis 2005;5:685-94.

4. Zühlke LJ, Steer AC. Estimates of the global burden of rheumatic heart disease. Glob Heart 2013;8:189-95.

5. Iung B, Baron G, Tornos P, Gohlke-Bärwolf C, Butchart EG, Vahanian A. Valvular heart disease in the community: a European experience. Curr Probl Cardiol 2007;32:609-61.

6. Unger P, Pibarot P, Tribouilloy C, Lancellotti P, Maisano F, Iung B, Piérard L; European Society of Cardiology Council on Valvular Heart Disease. Multiple and Mixed Valvular Heart Diseases. Circ Cardiovasc Imaging 2018;11:e007862.

7. Puntmann VO, Carr-White G, Jabbour A, Yu CY, Gebker R, Kelle S, Hinojar R, Doltra A, Varma N, Child N, Rogers T, Suna G, Arroyo Ucar E, Goodman B, Khan S, Dabir D, Herrmann E, Zeiher AM, Nagel E; International T1 Multicentre CMR Outcome Study. T1-Mapping and 
Outcome in Nonischemic Cardiomyopathy: All-Cause Mortality and Heart Failure. JACC Cardiovasc Imaging 2016;9:40-50.

8. Lee H, Park JB, Yoon YE, Park EA, Kim HK, Lee W, Kim YJ, Cho GY, Sohn DW, Greiser A, Lee SP. Noncontrast Myocardial T1 Mapping by Cardiac Magnetic Resonance Predicts Outcome in Patients With Aortic Stenosis. JACC Cardiovasc Imaging 2018;11:974-83.

9. Diao KY, Yang ZG, Xu HY, Liu X, Zhang Q, Shi K, Jiang L, Xie LJ, Wen LY, Guo YK. Histologic validation of myocardial fibrosis measured by T1 mapping: a systematic review and meta-analysis. J Cardiovasc Magn Reson 2016;18:92.

10. Taylor AJ, Salerno M, Dharmakumar R, Jerosch-Herold M. T1 Mapping: Basic Techniques and Clinical Applications. JACC Cardiovasc Imaging 2016;9:67-81.

11. Kim RJ, Fieno DS, Parrish TB, Harris K, Chen EL, Simonetti O, Bundy J, Finn JP, Klocke FJ, Judd RM. Relationship of MRI delayed contrast enhancement to irreversible injury, infarct age, and contractile function. Circulation 1999;100:1992-2002.

12. Aimo A, Gaggin HK, Barison A, Emdin M, Januzzi JL Jr. Imaging, Biomarker, and Clinical Predictors of Cardiac Remodeling in Heart Failure With Reduced Ejection Fraction. JACC Heart Fail 2019;7:782-94.

13. Reis Filho JR, Cardoso JN, Cardoso CM, PereiraBarretto AC. Reverse Cardiac Remodeling: A Marker of Better Prognosis in Heart Failure. Arq Bras Cardiol 2015;104:502-6.

14. Azevedo CF, Nigri M, Higuchi ML, Pomerantzeff PM, Spina GS, Sampaio RO, Tarasoutchi F, Grinberg M, Rochitte CE. Prognostic significance of myocardial fibrosis quantification by histopathology and magnetic resonance imaging in patients with severe aortic valve disease. J Am Coll Cardiol 2010;56:278-87.

15. Wong TC, Piehler KM, Kang IA, Kadakkal A, Kellman P, Schwartzman DS, Mulukutla SR, Simon MA, Shroff SG, Kuller LH, Schelbert EB. Myocardial extracellular volume fraction quantified by cardiovascular magnetic resonance is increased in diabetes and associated with mortality and incident heart failure admission. Eur Heart J 2014;35:657-64.

16. Olsen NT, Sogaard P, Larsson HB, Goetze JP, Jons C, Mogelvang R, Nielsen OW, Fritz-Hansen T. Speckletracking echocardiography for predicting outcome in chronic aortic regurgitation during conservative management and after surgery. JACC Cardiovasc Imaging 2011;4:223-30.
17. Legallois D, Hodzic A, Alexandre J, Dolladille C, Saloux E, Manrique A, Roule V, Labombarda F, Milliez P, Beygui F. Definition of left ventricular remodelling following ST-elevation myocardial infarction: a systematic review of cardiac magnetic resonance studies in the past decade. Heart Fail Rev 2020. [Epub ahead of print].

18. Hurst JW. The value of using the entire New York Heart Association's classification of heart and vascular disease. Clin Cardiol 2006;29:415-7.

19. Chopra P, Gulwani H. Pathology and pathogenesis of rheumatic heart disease. Indian J Pathol Microbiol 2007;50:685-97.

20. Shaper AG, Hutt MS, Coles RM. Necropsy study of endomyocardial fibrosis and rheumatic heart disease in Uganda 1950-1965. Br Heart J 1968;30:391-401.

21. Cunningham MW. Rheumatic fever, autoimmunity, and molecular mimicry: the streptococcal connection. Int Rev Immunol 2014;33:314-29.

22. González A, Schelbert EB, Díez J, Butler J. Myocardial Interstitial Fibrosis in Heart Failure: Biological and Translational Perspectives. J Am Coll Cardiol 2018;71:1696-706.

23. From AM, Maleszewski JJ, Rihal CS. Current status of endomyocardial biopsy. Mayo Clin Proc 2011;86:1095-102.

24. Monti CB, Zanardo M, Bosetti T, Alì M, De Benedictis E, Luporini A, Secchi F, Sardanelli F. Assessment of myocardial extracellular volume on body computed tomography in breast cancer patients treated with anthracyclines. Quant Imaging Med Surg 2020;10:934-44.

25. Dusenbery SM, Jerosch-Herold M, Rickers C, Colan SD, Geva T, Newburger JW, Powell AJ. Myocardial extracellular remodeling is associated with ventricular diastolic dysfunction in children and young adults with congenital aortic stenosis. J Am Coll Cardiol 2014;63:1778-85.

26. Everett RJ, Treibel TA, Fukui M, Lee H, Rigolli M, Singh A, et al. Extracellular Myocardial Volume in Patients With Aortic Stenosis. J Am Coll Cardiol 2020;75:304-16.

27. Mutnuru PC, Singh SN, D'Souza J, Perubhotla LM. Cardiac MR Imaging in the Evaluation of Rheumatic Valvular Heart Diseases. J Clin Diagn Res 2016;10:TC06-9.

28. Shriki J, Talkin B, Thomas IC, Farvid A, Colletti PM. Delayed gadolinium enhancement in the atrial wall: a novel finding in 3 patients with rheumatic heart disease. Tex Heart Inst J 2011;38:56-60.

29. Meel R, Nethononda R, Libhaber E, Dix-Peek T, Peters F, Essop M. Assessment of myocardial fibrosis by 
late gadolinium enhancement imaging and biomarkers of collagen metabolism in chronic rheumatic mitral regurgitation. Cardiovasc J Afr 2018;29:150-4.

30. Chaikriangkrai K, Lopez-Mattei JC, Lawrie G, Ibrahim H, Quinones MA, Zoghbi W, Little SH, Shah DJ.

Prognostic value of delayed enhancement cardiac magnetic resonance imaging in mitral valve repair. Ann Thorac Surg 2014;98:1557-63.

31. Zühlke L, Engel ME, Karthikeyan G, Rangarajan S, Mackie P, Cupido B, et al. Characteristics,

Cite this article as: Li S, Wang S, Yu J, Sun J, Cheng W, Liu J, Pu H, Chen Y, Peng L. Myocardial extracellular volume assessed by cardiovascular magnetic resonance may predict adverse left ventricular remodeling in rheumatic heart disease after valvular surgery. Quant Imaging Med Surg 2022;12(4):24872497. doi: 10.21037/qims-21-678 complications, and gaps in evidence-based interventions in rheumatic heart disease: the Global Rheumatic Heart Disease Registry (the REMEDY study). Eur Heart J 2015;36:1115-22a.

32. Remenyi B, ElGuindy A, Smith SC Jr, Yacoub M, Holmes DR Jr. Valvular aspects of rheumatic heart disease. Lancet 2016;387:1335-46.

33. Unger P, Rosenhek R, Dedobbeleer C, Berrebi A, Lancellotti P. Management of multiple valve disease. Heart 2011;97:272-7. 